\title{
Adjunct Therapy in Type 1 Diabetes: A Survey to Uncover Unmet Needs and Patient Preferences Beyond HbA1c Measures
}

\author{
Jeremy H. Pettus, MD, Jake A. Kushner, MD, ${ }^{2}$ Virginia Valentine, APRN-CNS, BC-ADM, CDE, FAADE, ${ }^{3}$ \\ Richard Wood, MBA, ${ }^{4}$ Christianne Pang, MBA, Sachin Paranjape, $\mathrm{PhD}^{5}$ Rachele Berria, MD, \\ Antonio Deluzio, DO, ${ }^{5}$ and Steven E. Edelman, MD ${ }^{6}$
}

\begin{abstract}
Background: Adjunct therapy can help patients with type 1 diabetes achieve glycemic goals while potentially mitigating some of the side effects of insulin. In this study, we used a patient survey to identify the unmet needs in type 1 diabetes therapy, patient views of treatment benefit-risk trade-offs, and patient preferences for the use of an adjunct therapy.

Methods: A quantitative survey was sent to 2084 adults with type 1 diabetes in November 2017. "Jobs-to-bedone" and conjoint analyses were performed on survey responses to identify unmet needs and the importance of treatment-associated benefits and risks to patients. A 5-point Likert scale measured the importance and satisfaction with patients' current therapy, and with gaps relating to unmet needs. In the conjoint analysis, patients were asked to choose between "packages" of attributes of two doses of adjunct therapy (200 and $400 \mathrm{mg}$ ) and placebo, based on established benefits and side effects.

Results: A total of 1313 patients $(63 \%)$ responded. The greatest unmet needs identified were simplifying treatment, lowering/maintaining glycated hemoglobin (HbA1c), reducing mental effort, and increasing time in range (TIR). Conjoint analysis showed that reductions in body weight and TIR had the highest attribute importance (25\% and $18 \%$, respectively). The majority (93\%) of patients had a preference for the adjunct therapy (either dose) over placebo.

Conclusions: This survey highlights the importance of measures beyond HbAlc, such as treatment simplification and TIR, and patient preference for adjunct therapies that help address unmet needs in type 1 diabetes treatment.
\end{abstract}

Keywords: Survey, Patient-reported outcomes, Treatment.

\section{Introduction}

$\mathbf{T}$ YPE 1 DIABETES affects not only glycemic control but also many aspects of a patient's life, including emotional well-being, quality of life, working ability, and social interactions. ${ }^{1-3}$ Patients with type 1 diabetes are dependent on insulin-replacement therapy, requiring multiple daily injections of insulin or a continuous subcutaneous insulin infusion pump. In addition, monitoring of blood glucose using multiple daily finger-stick measurements or continuous glucose monitoring (CGM) is recommended for better glycemic control. ${ }^{4}$ Developments in insulin therapy and technology

\footnotetext{
${ }^{1}$ University of California San Diego, La Jolla, California.

${ }^{2}$ McNair Interests, McNair Medical Institute, Houston, Texas.

${ }_{4}^{3}$ Clinica La Esperanza, Albuquerque, New Mexico.

${ }^{4}$ dQ\&A-The Diabetes Research Company, San Francisco, California.

${ }^{5}$ Sanofi US, Bridgewater, New Jersey.

${ }^{6}$ Veterans Affairs Medical Center, University of California San Diego, San Diego, California.

Prior Publication: Parts of this study were presented as a poster at AADE, August 17-20, 2018, Baltimore, MD; ADA RWE, November 16-18, 2018, Washington, DC; MEDS East, October 10-13, 2018, Orlando FL.

Valentine V, et al. Sotagliflozin as Adjunct Therapy in Type 1 Diabetes: A Survey to Uncover Unmet Needs and Patient Preferences.

(C) Jeremy H. Pettus, et al., 2019; Published by Mary Ann Liebert, Inc. This Open Access article is distributed under the terms of the Creative Commons Attribution Noncommercial License (http://creativecommons.org/licenses/by-nc/4.0/) which permits any noncommercial use, distribution, and reproduction in any medium, provided the original author(s) and the source are credited.
} 
over recent decades include pens, inhaled insulin, long-acting basal insulins (such as glargine $300 \mathrm{U} / \mathrm{mL}$ and degludec), faster-acting prandial insulins, pramlintide, pumps, and CGM. Despite these continued advances in management, particularly in the types of devices available, type 1 diabetes remains a constant daily burden for patients. Only an estimated one in five children and one in three adults with type 1 diabetes in the United States reach glycated hemoglobin (HbA1c) targets, ${ }^{5}$ and many still face issues with hypoglycemia. Real-world studies have estimated that most individuals with type 1 diabetes experience approximately two episodes of nonsevere hypoglycemia each week, and one severe event per patient per year. ${ }^{6}$ In addition, an estimated two-thirds of patients with type 1 diabetes are overweight or obese, leading to an increased risk of complications, such as hypertension, dyslipidemia, and cardiovascular disease, compared with healthy-weight individuals. $^{7-9}$

In recent years, other agents already approved for treatment of type 2 diabetes, such as metformin, glucagon-like peptide-1 receptor agonists, and sodium/glucose cotransporter (SGLT) inhibitors, have been investigated for their use as adjunct therapy to insulin for type 1 diabetes. ${ }^{4}$ The goals of adjunct therapy include helping patients with type 1 diabetes achieve glycemic targets, lowering the risk of hypoglycemia, reducing weight, improving quality of life, and increasing time in range (TIR). ${ }^{10}$ While adjunct therapies have shown beneficial effects in these various categories, some safety concerns remain. For example, SGLT inhibitors are associated with an increased risk of diabetic ketoacidosis (DKA) ${ }^{11}$; therefore, health care providers and patients need to weigh up the potential benefits and risks before considering these therapies in their treatment regimen.

When navigating the risk-benefit ratio, it is important to realize that patients' and health care providers' views may differ on the importance of individual risk and benefit factors. ${ }^{12}$ In general, there is a lack of data on how patients perceive the addition of adjunct therapies to their current regimens. Therefore, market research tools, such as "jobs-to-be-done" and conjoint analysis, are increasingly used to shed light on unmet needs and patient preferences, helping health care providers in the decision-making on the most appropriate treatments for their patients. ${ }^{13-15}$ In this article, we describe the outcomes of a patient survey, using the widely used "jobs-to-be-done" market research technique to uncover unmet needs (and thus identify jobs that need to be done) in the current type 1 diabetes therapy, and conjoint analysis to identify how patients viewed the trade-offs between benefits and side effects, and whether they would have a preference for use of an adjunct therapy.

\section{Research Design and Methods}

\section{Qualitative assessment before the survey}

Before the quantitative survey, and in accordance with best practice on the use of conjoint analysis for the study of patient preferences, ${ }^{16,17}$ two qualitative research exercises were used to build a deeper understanding of the patient experience and to select the most appropriate language for the questionnaire, by identifying the terms that patients were most familiar with. The first exercise was an online conversation with 50 participants from an existing online community, which took place over 3 days in October 2017 in the United States. The second exercise was a series of five live in-depth interviews with small groups of patients with type 1 diabetes (two or three per interview), carried out at a market research facility in October 2017 (New York). The online conversations were to assess the main burdens of diabetes at four different levels: (1) medical (e.g., day-to-day treatment and management of diabetes and related complications); (2) logistical (burden associated with the use of medical device and consumables); (3) financial (out-of-pocket costs, private insurance, etc.); and (4) emotional (stress associated with the disease); and interviews were assessed qualitatively and used to test and refine the terminology that would then be used in the quantitative survey.

\section{Patients and study design}

The quantitative survey was carried out in November 2017 by dQ\&A Market Research, Inc. The market research company sent an online survey to 2084 members with type 1 diabetes of its opt-in U.S.-based patient research panel for ongoing research on opinions of patients with diabetes. Patients included in the panel were recruited from established type 1 diabetes communities. The bona fide status of patients was confirmed by their responses in previous surveys. Patients included in the survey were diagnosed with type 1 diabetes; living in the United States; aged 18 years or older; and had recently completely one of the dQ\&A quarterly surveys which are sent regularly to all patients on the panel. Respondents received a nominal compensation (USD 10) for completing the survey, although this was not mentioned when they were initially recruited to the panel. The survey was blinded; the participants were not given any information on the study sponsor, product, or company names. The study sponsor was not given information on the identity of the participants.

\section{Quantitative survey analysis}

The survey had three distinct elements: characterization of the survey respondents; jobs-to-be-done analysis (identifying unmet needs); and conjoint analysis (assessment of patient preferences). In accordance with current market research best practice, all incomplete responses; surveys completed in an unrealistically short time; and all responses with randomly typed or nonsensical write-in responses to open-endquestions were removed, to produce a final validated data set. Statistical significance testing for all analyses of survey responses was performed at the $90 \%$ confidence level.

\section{Characterization of survey respondents}

Characterization of the survey respondents included collection of demographic data, self-reported diabetes outcomes, complications, comorbidities, estimated time (minutes/day) spent managing diabetes, height, weight, hypoglycemia awareness, use of CGM, and currently used insulin-delivery method. These characteristics were identified as important, based on responses from previous surveys, and updated by the respondents during the current survey.

\section{Unmet patients' needs analysis}

The jobs-to-be-done analysis is a widely used tool in market research to uncover and measure unmet needs. The survey included the following 15 outcomes that patients 
were least satisfied with from a list of 44 outcomes used in a previous dQ\&A survey ${ }^{18}$ : postprandial blood glucose $<180 \mathrm{mg} / \mathrm{dL}$; overnight blood glucose $70-130 \mathrm{mg} / \mathrm{dL}$; blood glucose during the day $70-180 \mathrm{mg} / \mathrm{dL}$; morning (fasting) blood glucose 70-180 mg/dL; HbA1c reduced or maintained at target; prevention of stress and anxiety caused by diabetes ("diabetes distress"); prevention of weight gain; prevention of hypoglycemic events not requiring medical assistance; prevention of severe hypoglycemic events requiring medical assistance; prevention of negative impact of diabetes on family and loved ones; general well-being throughout the day; simple and predictable diabetes management; reduced mental effort needed to manage diabetes; prevention of feelings of isolation and stigma due to diabetes; and flexibility with exercise and diet.

A five-level "Likert scale" was used to measure the importance (1 [not at all important] to 5 [extremely important]) and the degree of satisfaction (1 [not at all satisfied] to 5 [extremely satisfied]) of the outcomes from their current therapy. Importance and satisfaction scores were calculated as the percentage of respondents selecting the score of 4 or 5 on the Likert scale in importance and satisfaction of each outcome, respectively. This percentage was converted to a numerical score from 0 to 10 (with $100 \%$ being a score of 10).

To rank the unmet needs relative to each other, an "unmet needs score" was calculated based on the importance and satisfaction scores, and the "gap" between them, using the following formula:

Unmet needs score $=$ Importance score

$$
+ \text { (Importance score }- \text { Satisfaction score }) \text {. }
$$

The unmet needs score was used to identify the opportunities for improvement on the current treatment, with a score $>10$ indicating that needs are not currently being met effectively (higher scores indicate a bigger unmet need).

\section{Conjoint analysis of patient preference}

To uncover preferences and simulate the real-world decision-making process, that is, consideration of trade-offs between benefits and side effects, a discreet-choice research technique called conjoint analysis was used. This method is used to avoid asking separately about individual preferences for specific benefits or side effects, ${ }^{16,17}$ given that a rational response would be to seek to maximize all available benefits and reject all possible side effects. Using conjoint analysis, participants were presented with pairwise choices of "packages" of drug attributes (i.e., benefits or side effects) for selection. Each attribute was given various levels; for example, the effect on time in the ideal glucose range could be $20 \mathrm{~min}$ more, $1 \mathrm{~h}$ more, or $2.5 \mathrm{~h}$ more per day.

In this study, the packages considered by respondents were drawn from outcomes of clinical trials of an SGLT adjunct therapy (sotagliflozin). Participants were presented with nine pairwise choices of packages, each consisting of attributes drawn from the outcomes of the placebo (insulin only), the $200 \mathrm{mg}$, and the $400 \mathrm{mg}$ doses. Each package was made up of the following 10 attributes: reduction in $\mathrm{HbA} 1 \mathrm{c}$; reduction in rapid-acting insulin dose required for meals; increased time in the ideal blood glucose range; impact on weight; reduction in systolic blood pressure; impact on hypoglycemic events; risk of severe hypoglycemia; risk of DKA; risk of yeast infection; and risk of diarrhea. Different levels within each attribute representing the outcomes of the clinical trials were used as part of the conjoint analysis (see Supplementary Table S1). Note that levels and attributes can be positive (a benefit) or negative (a side effect). As part of the conjoint analysis, an underlying "utility" value was calculated for each level for each attribute. Utility scores were then summed to form specific packages that reflect the placebo, the $200 \mathrm{mg}$, and the $400 \mathrm{mg}$ doses. The utilities were summed to calculate the patient "preference share," which was used to compare respondent preference for the three packages analyzed (placebo, $200 \mathrm{mg}$ dose, and $400 \mathrm{mg}$ dose). The relative importance of individual attributes to the respondents (summing to $100 \%$ ) was also calculated as part of the analysis. Differences between importance scores were tested for statistical significance at the $90 \%$ confidence level and categorized into tiers, with no significant difference within tiers. Data were analyzed for all survey respondents, and for individual groups of respondents based on the characteristics gathered in the first part of the survey.

\section{Willingness to take an oral medication}

Participants' willingness to take an oral medication, in addition to optimized insulin therapy, was also analyzed. Survey participants were given a choice of "definitely," "likely," "unlikely," or "definitely not" to the question whether they would be willing to take a pill to help manage their diabetes. Responses were analyzed for the overall group, and based on the number of pills $(0,1-3$, or $\geq 4)$ per day the participants were taking at the time of the survey.

\section{Results}

\section{Characterization of patients and demographics}

In total, $1313(63 \%)$ of the 2084 participants, aged 20-86 years, who were contacted returned a valid completed survey. Baseline characteristics of the respondents are shown in Table 1 . Twenty percent of the respondents were aged $\geq 65$ years (Supplementary Fig. S1). The majority of respondents (78\%) used an insulin pump rather than multiple daily injections. Most of the CGM users also used an insulin pump (715/806; 89\%) (Supplementary Fig. S1). The frequency of self-monitored blood glucose tests was higher in non-CGM users (median 6 tests/day) compared with CGM users (median 4 tests/day) and ranged from not testing every day to $\geq 10$ tests per day in both groups. At the time of survey, $58 \%$ of respondents self-reported an HbA1c of $<7.0 \%(<53 \mathrm{mmol} /-$ $\mathrm{mol}), 31 \%$ had an $\mathrm{HbA} 1 \mathrm{c}$ between $7.0 \%$ (53 $\mathrm{mmol} / \mathrm{mol})$ and $8.0 \%(64 \mathrm{mmol} / \mathrm{mol})$, and $11 \%$ of respondents reported an $\mathrm{HbA} 1 \mathrm{c}$ of $>8.0 \%$ ( $>64 \mathrm{mmol} / \mathrm{mol}$ ). The time spent managing diabetes varied between CGM and non-CGM users, but no clear trends were observed by age group or in association with HbA1c or TIR (Supplementary Fig. S2).

\section{Unmet needs (jobs-to-be-done) analysis}

Twelve of the 15 outcomes had opportunity scores greater than 10 , indicating that these are significant unmet needs in type 1 diabetes with a large gap between importance and satisfaction (Fig. 1). Overall, the greatest opportunities for 
Table 1. Demographic Data and Clinical Characteristics of Survey Respondents

\begin{tabular}{|c|c|}
\hline Characteristics & Respondents $(\mathrm{n}=1313)$ \\
\hline $\begin{array}{l}\text { Female } \\
\text { Median age (range), years } \\
\text { 18-34 } \\
\text { 35-49 } \\
50-64 \\
\geq 65\end{array}$ & $\begin{aligned} & 880(67) \\
& 53(20-86) \\
& 155(12) \\
& 396(30) \\
& 497(38) \\
& 265(20)\end{aligned}$ \\
\hline $\begin{array}{l}\text { Median diabetes duration (range), years } \\
\text { Mean BMI, } \mathrm{kg} / \mathrm{m}^{2} \\
\text { HbA1c } \\
<7 \%(<53 \mathrm{mmol} / \mathrm{mol}) \\
7 \%-8 \%(53-64 \mathrm{mmol} / \mathrm{mol}) \\
>8 \%(>64 \mathrm{mmol} / \mathrm{mol})\end{array}$ & $\begin{array}{c}30(2-75) \\
27.4 \\
736(58) \\
401(31) \\
138(11)\end{array}$ \\
\hline $\begin{array}{l}\text { Time in ideal blood glucose range, } \% \\
0-19 \\
20-39 \\
40-59 \\
60-79 \\
80-100\end{array}$ & $\begin{array}{c}4 \\
15 \\
34 \\
33 \\
14\end{array}$ \\
\hline $\begin{array}{l}\text { Insulin delivery method } \\
\text { Pump } \\
\text { MDI }\end{array}$ & $\begin{array}{r}1023(78) \\
290(22)\end{array}$ \\
\hline $\begin{array}{l}\text { CGM use } \\
\text { Yes } \\
\text { No }\end{array}$ & $\begin{array}{l}806(61) \\
507(39)\end{array}$ \\
\hline $\begin{array}{l}\text { Most common comorbidities, } \%^{\mathrm{a}} \\
\text { High blood pressure } \\
\text { High cholesterol or triglycerides } \\
\text { Hypothyroidism or underactive thyroid } \\
\text { Depression } \\
\text { Diabetes-related eye problems }\end{array}$ & $\begin{array}{l}30 \\
30 \\
29 \\
17 \\
13\end{array}$ \\
\hline
\end{tabular}

Unless stated otherwise, data are expressed as number of patients $(\%)$.

${ }^{\text {a } I n c l u d e s ~ c o m o r b i d i t i e s ~ w i t h ~ a ~ p r e v a l e n c e ~ o f ~}>10 \%$.

BMI, body mass index; CGM, continuous glucose monitoring; HbA1c, glycated hemoglobin; MDI, multiple daily injections.

improving on current treatment in type 1 diabetes were in simplifying treatment (score of 13.6), lowering or maintaining $\mathrm{HbA1c}$ (score of 13.5), reducing mental effort (score of 13.2), and increasing TIR (70-180 mg/dL; score of 13.2).

Some variation across respondent groups was observed. For example, lowering/maintaining an HbA1c of $<7.0 \%$ (53 $\mathrm{mmol} / \mathrm{mol})$ was identified as the most important unmet need for the two groups of patients with an HbAlc above target $(7.0 \%-8.0 \%$ [53-64 $\mathrm{mmol} / \mathrm{mol}]$, and $>8.0 \%$ [>64 mmol/mol]) with scores of 15.4 and 18.0 , respectively. In contrast, in the group of respondents with an HbA1c of $<7.0 \%$ ( $53 \mathrm{mmol} / \mathrm{mol})$, lowering/maintaining $\mathrm{HbA} 1 \mathrm{c}$ received a score of only 11.5 (Supplementary Table S2). Preventing weight gain had a higher unmet needs score in the group that said they would like to lose weight (score of 15.9) compared with those who did not feel that they would like to lose weight (score of 2.3) (Supplementary Table S2). On average, CGM users reported higher unmet needs scores than those not using CGM. For CGM users, the highest unmet needs scores were for treatment simplification (14.1 vs. 12.7 of non-CGM users), reducing mental effort, and improving TIR, whereas for non-CGM users, the greatest unmet need was lowering or maintaining HbA1c (Supplementary Table S2).

\section{Conjoint analysis}

Across all respondents, weight reduction had the highest attribute importance (25\%), followed by TIR of blood glucose 70-180 mg/dL (18\%) (Fig. 2). Reduction in HbA1c and yeast infections was ranked third in attribute importance (12\% and $11 \%$, respectively), followed by diarrhea (9\%) and DKA $(7 \%)$. Hypoglycemia was ranked together with a reduction in blood pressure as least important, with only $3 \%$ importance value. The results were generally consistent across all respondent subgroups (Supplementary Table S3). However, the risk of yeast infection was statistically less important to male than female respondents (9\% vs. $12 \%$ ). Among respondents who reported a need to lose weight, change in weight had the highest importance $(27 \%)$, whereas weight change was significantly less important $(18 \%)$ to respondents who reported not having the need to lose weight.

The analysis also showed that the majority of respondents (93\%) preferred the addition of the adjunct therapy (200 or $400 \mathrm{mg}$ ) over the placebo. Considered individually, the $200 \mathrm{mg}$ dose had higher shares of preference $(93 \%)$ than the $400 \mathrm{mg}$ dose $(86 \%)$ when compared with placebo (insulinonly therapy) (Fig. 3). However, when the 200, $400 \mathrm{mg}$, and placebo doses were compared at the same time, $73 \%$ of 


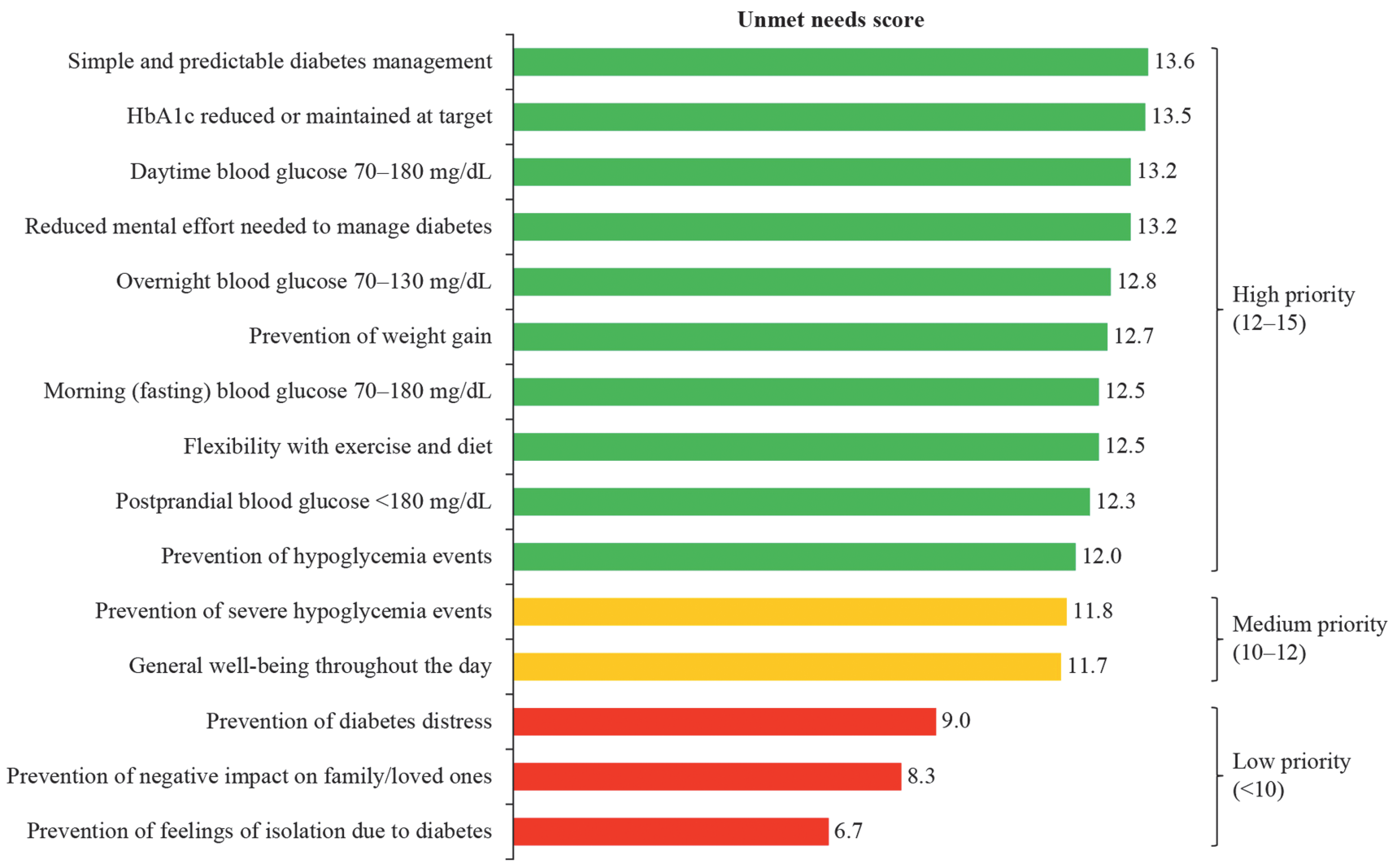

FIG. 1. Jobs-to-be-done opportunity scores for each of the 15 unmet needs analyzed. Unmet needs score was calculated as follows: Importance score + (Importance score - Satisfaction score). Unmet needs scores $\geq 10$ indicate important needs that are not being met effectively or at all and scores $<10$ indicate needs that either are not important or being met effectively. HbA1c, glycated hemoglobin.

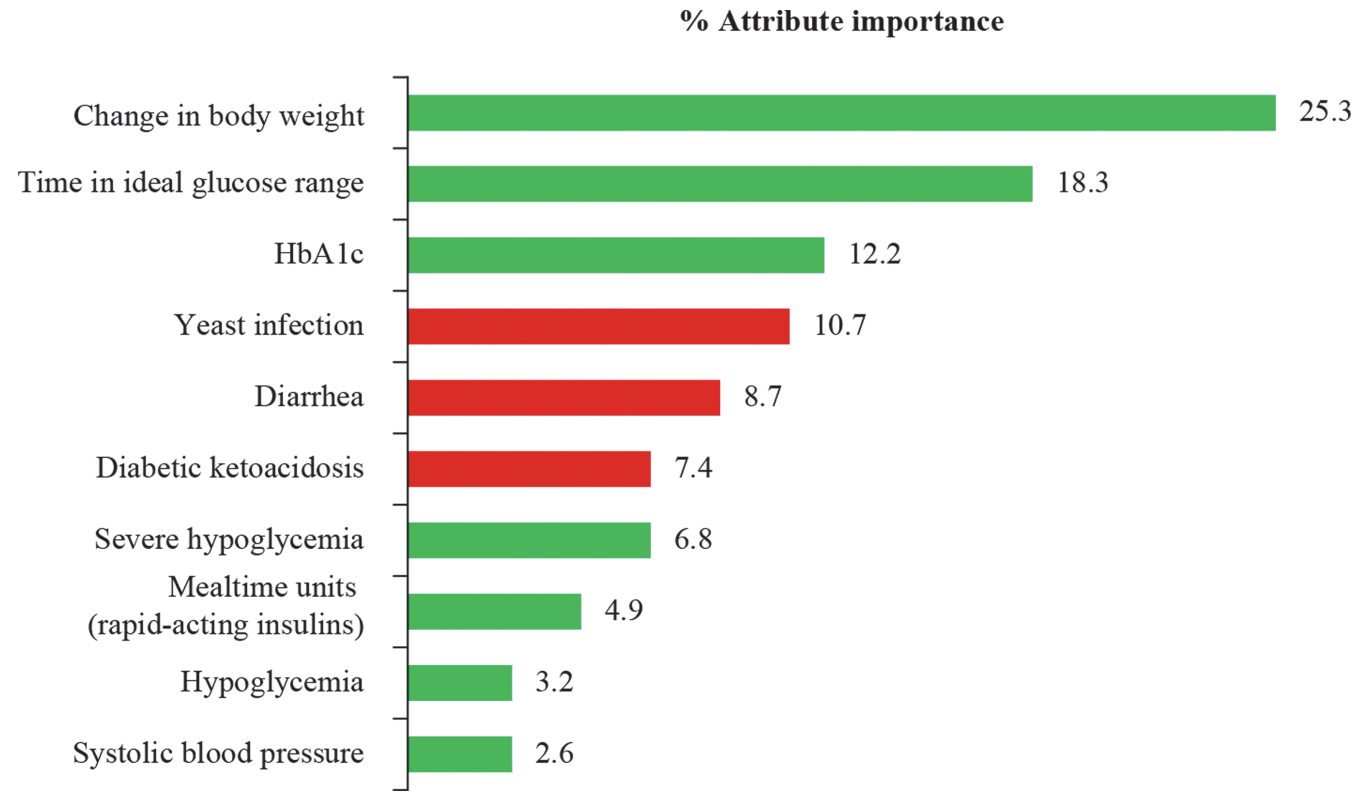

FIG. 2. Most important attributes from conjoint analysis $(n=1313)$. Green and red segments represent benefits and side effects of sotagliflozin, respectively. HbA1c, glycated hemoglobin. 

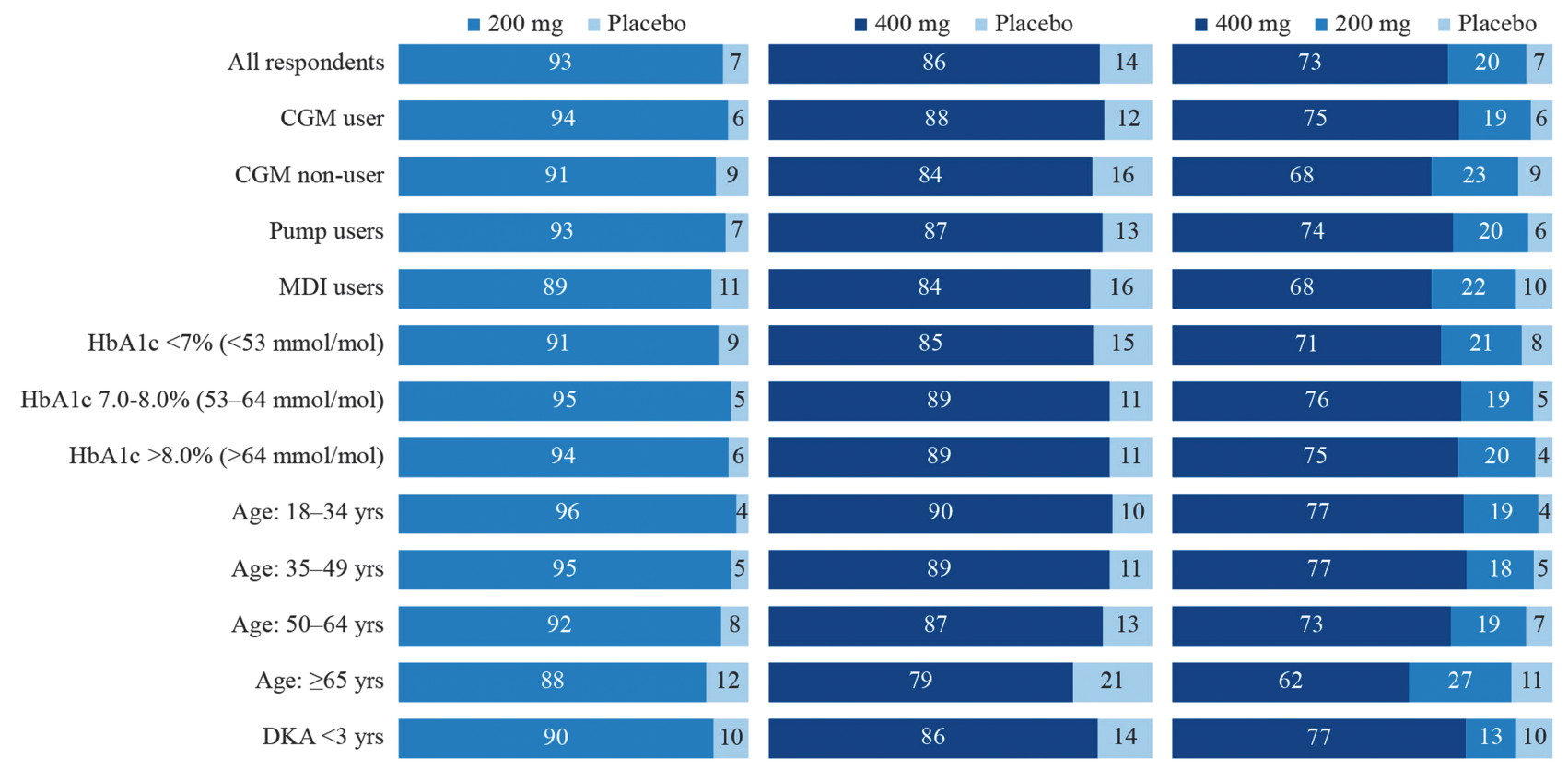

FIG. 3. Comparison of preferences of respondents for the $200 \mathrm{mg}$ dose adjunct therapy versus placebo, the $400 \mathrm{mg}$ adjunct therapy versus placebo, and for all three packages. CGM, continuous glucose monitoring; DKA, diabetic ketoacidosis; HbA1c, glycated hemoglobin; MDI, multiple daily injections.

respondents indicated a preference for the $400 \mathrm{mg}$ dose, with the $200 \mathrm{mg}$ dose receiving $20 \%$ preference share (Fig. 3).

\section{Willingness to take oral medication}

In total, $77 \%$ of respondents indicated that they were already taking at least one oral medication daily in addition to insulin therapy at the time of the survey. Significantly more patients already taking one or more pills per day (any medication) indicated that they "definitely" would take a pill to help better manage their diabetes compared with those not taking any pills with their current therapy (Supplementary Table S4). Similarly, a higher percentage of participants not taking pills at the time of the survey indicated that they were "unlikely" or "definitely would not" take pills (16\%) compared with those who are already taking 1-3 pills/day $(9 \%)$ or $\geq 4$ pills/day (6\%) (Supplementary Table S4).

\section{Discussion}

These analyses identified 12 needs that are not adequately met by current treatment for patients with type 1 diabetes. Not surprisingly, HbA1c measures ranked highly in both the unmet needs analysis and the conjoint analysis. Interestingly though, many of the most important needs and preferences identified were measures beyond HbA1c, such as improvement in TIR, weight reduction, and reducing mental effort. The results of this survey highlight patients' desire to simplify management of type 1 diabetes treatment, prevent weight gain (a common problem with insulin therapy), and improve or achieve greater TIR. Overall, 93\% of respondents would consider adding the adjunct therapy to insulin to help address some of these unmet needs.

While the majority of attributes were similar between the different patient groups, some opportunities for improvement on the currently available treatment varied in importance across patient groups. As expected, reducing HbAlc was much more important in the groups not at $\mathrm{HbAlc}$ target (i.e., those with an $\mathrm{HbA} 1 \mathrm{c}$ of $>7.0 \%$ [ $>53 \mathrm{mmol} / \mathrm{mol}]$ ) than those who were already at target $\mathrm{HbA} 1 \mathrm{c}$ levels. Similarly, preventing weight gain/losing weight was much more important to those respondents who would like to lose some weight than to those who did not feel that they needed to. Interestingly, prevention of hypoglycemia and severe hypoglycemia was identified as lower priority unmet needs. This may be related to the high percentage of respondents who were CGM users, as they may feel less concerned about hypoglycemia risk due to low blood glucose alerts and alarms provided by the device. DKA was given a low importance score, despite its potentially serious consequences. The survey outcomes suggest that while there is some level of awareness around this issue, the majority (69\%) of respondents had not experienced DKA since being diagnosed and 30\% did not test ketones on a regular basis (data not shown), which is similar to findings from other questionnaires. ${ }^{19}$ The lower importance of both hypoglycemia risk and DKA also highlights a possible educational gap in patients with type 1 diabetes, which might help to improve their diabetes management, glycemic variability, and overall well-being. The education of patients about DKA may be especially important for those who have SGLT2s added to their treatment regimens, given their potential association with increased rates of DKA. ${ }^{20}$

Conjoint analysis also indicated that change in body weight and TIR were considered the most important drivers of patient preference, as with the jobs-to-be-done opportunity scoring. All respondents were concerned about TIR, regardless of whether they were CGM users or not. However, pump users were also more concerned about TIR than patients who used multiple daily injections. Overall, simplification of treatment was considered the greatest opportunity for improvement on current treatments, even in the groups of patients who used insulin pumps and CGM. In agreement with the drivers of patient preference identified in the conjoint 
analysis, the survey respondents had a strong preference for use of the adjunct therapy with sotagliflozin (after evaluation of the risk-benefit profile, more than $90 \%$ of respondents would consider use of the adjunct therapy), which has demonstrated weight loss and increased TIR in patients with type 1 diabetes, despite the increased risk of DKA. ${ }^{21-23}$

There were a number of limitations to this study. First, the outcomes (benefits and risks) considered were taken from clinical trials, rather than real-world evidence, and therefore may not be completely representative of clinical practice experience. Second, as mentioned above, more than half the patients included had achieved target HbA1c levels, which is considerably higher than the estimates of $14 \%-30 \%$ from public registries. ${ }^{24}$ This may limit the direct application of the results to the broader U.S. population of patients with type 1 diabetes. In addition, the higher proportion of patients already at target and over 65 years of age may have reduced the importance of $\mathrm{HbA} 1 \mathrm{c}$ reduction and hypoglycemia risk compared with younger patients and those with poor glycemic control. Finally, as these data were obtained from an e-mail survey, the results may be subject to self-selection bias. Despite these limitations, the high response rate to the survey and consistency in responses indicated that many patients with type 1 diabetes are not satisfied with their current treatment options and are willing to consider adjunct therapies, despite the potential increased risks of these therapies.

\section{Conclusions}

This survey highlights that there are still many unmet needs in type 1 diabetes, including achievement of glycemic control targets (HbA1c $<7 \%$ [ $<53 \mathrm{mmol} / \mathrm{mol}]$ ), prevention of weight gain, increased time spent in the optimal blood glucose range, and a desire for simpler diabetes management. The benefits of new adjunct therapies appear to be aligned with some of these needs, as more than $90 \%$ of respondents would consider taking the medication evaluated in this survey, after appraising its risk-benefit profile.

\section{Acknowledgments}

This analysis was funded by Sanofi US, Inc. The authors received writing/editorial support in the preparation of this article provided by Michael van der Veer, $\mathrm{PhD}$, of Excerpta Medica, funded by Sanofi US, Inc.

\section{Authors' Contributions}

J.H.P., J.A.K., V.V., A.D., and S.E. were involved in data analysis and interpretation, provided critical revisions, and gave approval of the final draft. R.W. and C.P. were involved in the concept and design of the study, data acquisition, data analysis and interpretation, provided critical revisions, and gave approval of the final draft. S.P. and R.B. were involved in the concept and design of the study, data analysis and interpretation, provided critical revisions, and gave approval of the final draft.

\section{Author Disclosure Statement}

J.H.P. serves or has served on the advisory boards for Insulet, MannKind, Novo Nordisk, Sanofi, Tandem Diabetes, and Valeritas; and is a member of the speakers' bureau for Sanofi and Valeritas. J.A.K. serves or has served on the ad- visory boards for Lexicon and Sanofi; and is a full-time employee of McNair Interests, a private equity group. V.V. serves on the advisory boards for Abbott Diabetes Care, AstraZeneca, CeQur, Eli Lilly, Intarcia, Janssen, and Novo Nordisk; is a consultant for Abbott Diabetes Care, Adocia, CeQur, and Eli Lilly; and a speaker for Abbott Diabetes Care, AstraZeneca, Eli Lilly, and Janssen. R.W. is CEO of dQ\&A Market Research, Inc. C.P. is Vice-President of Research and Analytics, dQ\&A Market Research, Inc. S.P., R.B., and A.D. are employees of Sanofi US, Inc. S.E. serves on advisory boards for Lilly, Novo Nordisk, and Sanofi.

\section{Supplementary Material}

Supplementary Figure S1

Supplementary Figure S2

Supplementary Table S1

Supplementary Table S2

Supplementary Table S3

Supplementary Table S4

\section{References}

1. Ducat L, Rubenstein A, Philipson LH, et al.: A review of the mental health issues of diabetes conference. Diabetes Care 2015;38:333-338.

2. Rydén A, Sörstadius E, Bergenheim K, et al.: The humanistic burden of type 1 diabetes mellitus in Europe: examining health outcomes and the role of complications. PLoS One 2016;11:e0164977.

3. Vanstone M, Rewegan A, Brundisini F, et al.: Patient perspectives on quality of life with uncontrolled type 1 diabetes mellitus: a systematic review and qualitative metasynthesis. Ont Health Technol Assess Ser 2015;15:1-29.

4. American Diabetes Association (ADA): Standards of medical care in diabetes-2018. Diabetes Care 2018;41:S1-S159.

5. Agiostratidou G, Anhalt H, Ball D, et al.: Standardizing clinically meaningful outcome measures beyond $\mathrm{HbA}_{1 \mathrm{c}}$ for type 1 diabetes: a consensus report of the American Association of Clinical Endocrinologists, the American Association of Diabetes Educators, the American Diabetes Association, the Endocrine Society, JDRF International, The Leona M. and Harry B. Helmsley Charitable Trust, the Pediatric Endocrine Society, and the T1D Exchange. Diabetes Care 2017;40:1622-1630.

6. Elliott L, Fidler C, Ditchfield A, et al.: Hypoglycemia event rates: a comparison between real-world data and randomized controlled trial populations in insulin-treated diabetes. Diabetes Ther 2016;7:45-60.

7. Conway B, Miller RG, Costacou et al.: Temporal patterns in overweight and obesity in type 1 diabetes. Diabet Med 2010;27:398-404.

8. de Ferranti SD, de Boer IH, Fonseca V, et al.: Type 1 diabetes mellitus and cardiovascular disease: a scientific statement from the American Heart Association and American Diabetes Association. Diabetes Care 2014;37:2843-2863.

9. Redondo MJ, Foster NC, Libman IM, et al.: Prevalence of cardiovascular risk factors in youth with type 1 diabetes and elevated body mass index. Acta Diabetol 2016;53:271-277.

10. Bode BW, Garg SK: The emerging role of adjunctive noninsulin antihyperglycemic therapy in the management of type 1 diabetes. Endocr Pract 2016;22:220-230.

11. Harris K, Boland C, Meade L, et al.: Adjunctive therapy for glucose control in patients with type 1 diabetes. Diabetes Metab Syndr Obes 2018;11:159-173. 
12. Reed Johnson F, Zhou M: Patient preferences in regulatory benefit-risk assessments: a US perspective. Value Health 2016;19:741-745.

13. Hofheinz R, Clouth J, Borchardt-Wagner J, et al.: Patient preferences for palliative treatment of locally advanced or metastatic gastric cancer and adenocarcinoma of the gastroesophageal junction: a choice-based conjoint analysis study from Germany. BMC Cancer 2016;16:937.

14. Louder AM, Singh A, Saverno K, et al.: Patient preferences regarding rheumatoid arthritis therapies: a conjoint analysis. Am Health Drug Benefits 2016;9:84-93.

15. Ryan M, Farrar S: Using conjoint analysis to elicit preferences for health care. BMJ 2000;320:1530-1533.

16. Bridges JF, Hauber AB, Marshall D, et al.: Conjoint analysis applications in health-a checklist: a report of the ISPOR Good Research Practices for Conjoint Analysis Task Force. Value Health 2011;14:403-413.

17. Reed Johnson F, Lancsar E, Marshall D, et al.: Constructing experimental designs for discrete-choice experiments: report of the ISPOR Conjoint Analysis Experimental Design Good Research Practices Task Force. Value Health 2013; $16: 3-13$

18. Gopisetty D, Levine B, Liu N, et al.: How does diabetes affect daily life? A beyond-A1C perspective on unmet needs. Clin Diabetes 2018;36:133-137.

19. Albanese-O'Neill A, Wu M, Miller KM, et al.: Poor adherence to ketone testing in patients with type 1 diabetes. Diabetes Care 2017;40:e38-e39.
20. Chen J, Fan F, Wang JY, et al.: The efficacy and safety of SGLT2 inhibitors for adjunctive treatment of type 1 diabetes: a systematic review and meta-analysis. Sci Rep 2017;7:44128.

21. Buse JB, Garg SK, Rosenstock J, et al.: Sotagliflozin in combination with optimized insulin therapy in adults with type 1 diabetes: the North American inTandem1 Study. Diabetes Care 2018;41:1970-1980.

22. Danne T, Cariou B, Banks P, et al.: HbA1c and hypoglycemia reductions at 24 and 52 weeks with sotagliflozin in combination with insulin in adults with type 1 diabetes: the European inTandem2 Study. Diabetes Care 2018;41:19811990.

23. Garg SK, Henry RR, Banks P, et al.: Effects of sotagliflozin added to insulin in patients with type 1 diabetes. $\mathrm{N}$ Engl $\mathrm{J}$ Med 2017;377:2337-2348.

24. Miller KM, Foster NC, Beck RW, et al.: Current state of type 1 diabetes treatment in the U.S.: updated data from the T1D exchange clinic registry. Diabetes Care 2015;38: 971-978.

Address correspondence to:

Jeremy H. Pettus, MD

University of California San Diego

9500 Gilman Drive \#9111-G

La Jolla, CA 92093

E-mail:.jpettus@ucsd.edu 